\title{
刈割和施肥对高寒草甸功能多样性与地上净初级 生产力关系的影响
}

\author{
潘石玉 ${ }^{1}$ 孔涁涁 ${ }^{1}$ 姚天华 1 卫欣华 ${ }^{1}$ 李英年 $^{2}$ 朱志红 $^{{ }^{*}}$ \\ ${ }^{1}$ 陕西师范大学生命科学学院, 西安 710119; ${ }^{2}$ 中国科学院西北高原生物研究所, 西宁 810008
}

摘 要 功能多样性 $(F D)$ 对群落地上净初级生产力 $\left(A N P P, \mathrm{~g} \cdot \mathrm{m}^{-2} \cdot \mathrm{a}^{-1}\right)$ 有直接的影响, 但不同扰动因素对 $F D-A N P P$ 关系将产生 何种影响尚不明确。通过在青藏高原高寒矮生嵩草(Kobresia humilis)草甸连续7年(2007-2013)的刚割(3水平: 不刏割、留茬3 $\mathrm{cm}$ 的中度刈割和留茬 $1 \mathrm{~cm}$ 的重度刈割)和施肥(2水平: 施用 $12.75 \mathrm{~g} \cdot \mathrm{m}^{-2} \cdot \mathrm{a}^{-1}$ 尿素 $+3.06 \mathrm{~g} \cdot \mathrm{m}^{-2} \cdot \mathrm{a}^{-1}\left(\mathrm{NH}_{4}\right)_{2} \mathrm{HPO}_{4}$ 、不施肥)控制实验, 研究了刈割、施肥、年份及其交互作用对 $A N P P$ 和 $F D$ 的影响以及 FD-ANPP关系的变化。研究结果显示: (1)增加刈割强度和土 壤养分均显著提高了 $A N P P$, 但其效应随着年份的不同而有差异, ANPP随着时间呈波动下降趋势, 而FD则随年度增大; (2)施 肥显著增加了 $F D$, 而刚割对 $F D$ 无显著影响 $(p>0.05)$; (3)在6种刈割和施肥处理组合下, $F D-A N P P$ 关系随时间的变化主要受刈 割扰动影响呈现正线性相关和无相关两种模式。无论施肥与否, 不刚割(C0)群落中 FD和 $A N P P$ 之间无相关 $(n=90, p>0.05)$; 而刚割群落中 $F D$ 和 $A N P P$ 之间为正相关 $(n=90, p<0.05)$ 。由于刈割同时提高了 $F D$ 和 $A N P P$, 促使二者在刈割群落中呈现正线 性相关。(4)尽管施肥不影响 $F D$ 和 $A N P P$ 的关系模式, 但与刈割共同作用, 影响 $F D-A N P P$ 关系的截距或斜率。上述结果说明, 刈 割扰动和土壤营养资源共同影响着FD-ANPP关系, 而刈割在改变其关系模式方面起主导作用。

关键词 功能多样性, 地上净初级生产力, 刏割, 施肥

引用格式: 潘石玉, 孔涁涁, 姚天华, 卫欣华, 李英年, 朱志红 (2015). 刈割和施肥对高寒草甸功能多样性与地上净初级生产力关系的影响. 植物生态 学报, 39, 867-877. doi: 10.17521/cjpe.2015.0083

\section{Effects of clipping and fertilizing on the relationship between functional diversity and above- ground net primary productivity in an alpine meadow}

PAN Shi-Yu ${ }^{1}$, KONG Bin-Bin ${ }^{1}$, YAO Tian-Hua ${ }^{1}$, WEI Xin-Hua ${ }^{1}$, LI Ying-Nian ${ }^{2}$, and ZHU Zhi-Hong ${ }^{1 *}$

${ }^{1}$ College of Life Sciences, Shaanxi Normal University, Xi'an 710119, China; and ${ }^{2}$ Northwest Institute of Plateau Biology, Chinese Academy of Sciences, Xining 810008, China

\section{Abstract}

Aims Much attention has been paid for the relationship between species diversity (SD) and aboveground net primary productivity (ANPP). However, the effect of functional diversity (FD) on ANPP was more straightforward. Most researchers considered $S D$ or $F D$ as an independent variable to study the relationship between biodiversity and ANPP. In fact, the relationship may be affected by abiotic factors. Our objective was to study how different disturbance factors affect the relationship of FD-ANPP.

Methods The experiment was conducted in the alpine Kobresia humilis meadow at Haibei Research Station of the Chinese Academy of Sciences with clipping (unclipping, stubbled $3 \mathrm{~cm}$ and $1 \mathrm{~cm}$ ) and fertilizing (12.75 $\mathrm{g} \cdot \mathrm{m}^{-2} \cdot \mathrm{a}^{-1}$ urea $\left.+3.06 \mathrm{~g} \cdot \mathrm{m}^{-2} \cdot \mathrm{a}^{-1}\left(\mathrm{NH}_{4}\right)_{2} \mathrm{HPO}_{4}\right)$ treatments from 2007 to 2013. General linear model univariate ANOVA, regression analysis and ANCOVA were used for studying the effects of clipping, fertilizing, year and their interaction on ANPP and FD and the change of relationship of FD-ANPP.

Important findings ANPP was enhanced by increasing clipping intensity and soil nutrient extremely significantly $(p<0.01)$. However, the effect extent was different in each year. ANPP presented a fluctuant downward trend year-to-year while FD increased along time. FD was increased by fertilizing significantly $(p<0.05)$, while the effect of clipping on FD was not significant $(p>0.05)$. Under the six kinds of treatment combinations of clipping and fertilizing, the relationship of FD-ANPP presented two patterns including linear positive correlation and no correlation mainly depending on clipping. No matter the community was fertilized or not, in the unclipped

收稿日期Received: 2015-05-25 接受日期Accepted: 2015-08-13

* 通讯作者Author for correspondence (E-mail: zhuzhihong@snnu.edu.cn) 
community, FD showed no correlation with ANPP ( $n=90, p>0.05)$. However, in the clipped community, clipping increased $F D$ and $A N P P$ in the same time, which facilitated them showing linear positive correlation. Fertilizing cannot affect the pattern of relationship of $F D-A N P P$, but the slope and intercept of equations of relationship were influenced by fertilizing and clipping. The results suggested that the relationship of FD-ANPP was influenced by clipping and fertilizing jointly, while clipping plays a leading role in changing their relationship patterns. Key words functional diversity, aboveground net primary productivity, clipping, fertilizing

Citation: Pan SY, Kong BB, Yao TH, Wei XH, Li YN, Zhu ZH (2015). Effects of clipping and fertilizing on the relationship between functional diversity and aboveground net primary productivity in an alpine meadow. Chinese Journal of Plant Ecology, 39, $867-877$. doi: 10.17521/cjpe. 2015.0083

近年来，随着全球生物多样性的快速降低，生 物多样性与生态系统功能的关系成为生态学领域备 受关注的科学问题(Chapin et al., 2000)。生态系统生 产力是反映生态系统功能的最为综合的指标(如碳 氮循环、枯落物分解、动物采食等)(Chapin et al., 2000; Naeem, 2002), 植物群落的生产力则是生态系 统生产力的基础, 研究群落生产力对生物多样性变 化的响应对衡量生态系统功能具有重要意义 (Tilman et al., 1996)。

在生物多样性对植物群落生产力影响的研究中, 大多数学者仅强调了物种多样性或物种数目的作用, 物种多样性与群落生产力之间表现为驼峰型关系 (Kaiser，2000)或正相关关系(王长庭等, 2005; 江小 雷和张卫国, 2010)。但实际上, 不同物种在生理、 生态或形态学特征上存在很大差异(Lepš et al., 2001), 因而仅考虑物种丰富度和物种相对多度的 物种多样性指标很难反映物种属性对生态系统过程 的重要作用。很多研究发现, 植物功能多样性 $(F D)$ 对群落生产力有更为直接的影响(Díaz \& Cabido, 2001; Petchey \& Gaston, 2002; Bernhardt-Römermann et al., 2011)。功能多样性是指影响生态系统功 能过程的种间功能特征变化的范围, 即种间平均差 异(Tilman et al., 2001), 包含群落种间功能性状、物 种组成及其相对多度等基本成分的差异(Lepš et al., 2006)。理论研究表明, 功能多样性主要通过种间生 态位互补效应(niche complementarity effect)影响群 落的生产力, 即随着物种多样性增加, 群落中植物 性状(plant traits)(如根系深度、冠层高度、生长速度、 竞争能力及对不良环境的耐受力等)的多样化程度 也在增加, 实现了在不同时空、以不同方式对有限 资源的最大化利用, 减少了资源浪费, 从而使生产 力达到较高水平(江小雷和张卫国, 2010)。由于功能 多样性将有机体的功能属性与生态系统过程紧密相
连(Petchey \& Gaston, 2006), 并且可通过多个性状 描述不同的生态系统功能(Schleuter et al., 2010), 因 而对生态系统功能变化具有更准确的预测能力 (Mokany et al., 2008)。当前有关物种多样性和生态 系统功能关系的几乎全部假说(均衡效应除外)实质 上都依赖于种间性状差异(Hillebrand \& Matthiessen, 2009)。Tilman等(1997, 2001)的研究发现：物种丰富 度、功能群多样性和功能组成对生态系统过程都有 影响, 而功能组成和功能多样性占主导作用。Díaz 和Cabido (2001)对过去25项研究结果的分析表明, 功能组成和功能丰富度对生态系统过程的影响大于 物种多样性的影响。但也有研究认为功能多样性并 不必然和群落初级生产力直接相关, 因为在给定的 功能多样性条件下, 物种也可以通过各种性状组合 实现对资源的互补性利用而影响生产力(Hooper, 1998)。对高寒草甸的研究显示, 施肥对比叶面积和 比叶质量等单一性状的功能多样性有显著影响, 但 对多性状组合的功能多样性影响微弱(李晓刚等, 2011)。目前的研究表明, 功能多样性和生产力之间 可表现出负相关(Thompson et al., 2005; 藏岳铭等, 2009)、无相关(Griffin et al., 2009)或正相关(吕亭亭 等, 2014)等多种关系形式, 而且依赖于土壤营养状 况和群落所受的干扰强度(Bernhardt-Römermann et al., 2011; 李晓刚等, 2011), 说明两者间关系形式的 差异还与群落环境和扰动因素有密切联系。因此, 当同一群落长期遭受不同扰动因素的作用而进入不 同的群落状态后, 研究不同状态的群落在时间序列 上的功能多样性与生产力关系, 对于揭示功能多样 性变化对生产力的潜在影响具有重要意义。

高寒草甸生态系统作为青藏高原最重要的自然 生态系统类型之一, 是全球变化的敏感区和生物多 样性重点保护区。高寒矮生嵩草(Kobresia humilis) 草甸长期受家畜放牧的强烈影响, 特别是过度放牧 
常常导致其退化。由于地处高寒地区，虽然其土壤 有机质丰富, 但矿化率较低, 可利用养分缺乏, 不 能满足植物生长需要(赵新全, 2009), 施用化肥就成 为高寒草甸改良和管理中的常见措施。因此, 放牧 扰动强度和土壤养分获得性是影响该生态系统最重 要的两种人为因素。但长期施肥在提高该草地生产 力的同时, 也会降低物种丰富度(Yang et al., 2011), 而且刈割和施肥能分别增加和减小其功能多样性 (李晓刚等, 2011), 也同时分别降低和增加其地上净 初级生产力 (ANPP, $\left.\mathrm{g} \cdot \mathrm{m}^{-2} \cdot \mathrm{a}^{-1}\right)$ (王海东等, 2013)。本研 究试图通过在青藏高原高寒矮生嵩草草甸连续7年 (2007-2013)的刈割(3水平)和施肥(2水平)控制实验, 探讨在刚割与施肥处理组合作用下形成的6种处理 群落间功能多样性与地上净初级生产力关系的变 化。该研究群落在2007年以前处于家畜自由放牧状 态, 放牧强度中等, 无化肥施用。通过连续7年的实 验处理, 群落已在物种组成、物种多样性和生产力 等方面发生分异, 但其功能多样性和生产力之间的 关系是否随之发生变化仍不明确。由于功能多样性 和群落生产力关系依赖于土壤营养和扰动因素 (Hooper, 1998; Bernhardt-Römermann et al., 2011; 李晓刚等, 2011), 因此, 本文主要探讨以下问题: (1) 长期刚割和施肥对地上净初级生产力和功能多样性 有何影响? (2) 6种处理群落的地上净初级生产力和 功能多样性间的关系模式是否不同? (3)刚割和施 肥对此关系模式的变化有何影响?

\section{1 研究样地概况和方法}

\section{1 研究样地概况}

本实验在青海海北高寒草地生态系统国家野外 科学观测研究站的矮生嵩草草甸内进行。该站地理 坐标为 $37.48^{\circ}-37.75^{\circ} \mathrm{N}, 101.20^{\circ}-101.38^{\circ} \mathrm{E}$, 海拔 $3200 \mathrm{~m}$ 。研究区空气稀薄, 年降水量560 mm, 6-9 月降水量占全年降水量的 $68.6 \%$ 。研究区属于高寒地 区, 年平均气温 $-1.6{ }^{\circ} \mathrm{C}$, 全年无绝对无霜期, 相对无 霜期约为 20 天, 年日照时间可达 $2467.7 \mathrm{~h}$ 。样地土壤 为高山草甸土, 有机质分解缓慢, 土壤 $\mathrm{pH}$ 值为 6.0-7.5 (李英年等, 2004)。矮生嵩草草甸地势平坦, 是当地的冬春草场, 每年11月至次年6月初放牧, 6月 中上旬至 10 月底转至夏秋草场放牧, 家畜为藏羊和 牦牛。主要植物有矮生嵩草、垂穗披碱草(Elymus
nutans)、粗嗙薹草(Carex scabrirostris)、异针茅(Stipa aliena)、羊茅(Festuca ovina)、美丽风毛菊(Saussurea superba)、瑞苓草(S.nigrescens)、圆葶藦苓草(Morina chinensis)、蕨麻(Potentilla anserina)、雪白委陵菜 $(P$. nivea)、甘肃棘豆(Oxytropis kansuensis)、针裂银莲花 (Anemone obtusiloba) 和萎软紫苑(Aster flaccidus)等。

\section{2 研究方法}

\subsection{1 实验设计}

研究样地 $(100 \mathrm{~m} \times 60 \mathrm{~m})$ 用网围栏建于 2007 年4 月底。实验为 2 因子裂区设计, 包含 3 个刈割水平, 2 个施肥水平, 3 区组, 每区组 3 个大样方 $(4 \mathrm{~m} \times 4 \mathrm{~m})$ 。 主区为刈割处理(clipping treatment), 刈割留茬高度 分别为 $1 \mathrm{~cm}(\mathrm{C} 2) 、 3 \mathrm{~cm}(\mathrm{C} 1)$ 和不刏割(C0), 刈割量 分别占当年地上总生物量的 $60 \%-70 \%$ 、 $45 \%-50 \%$ 和 0, 刏割收获的生物量略高于该草地类型家畜放牧 强度实验中(朱志红等, 1994; 朱志红和王刚, 1996; Zhu et al., 2012)重度和中度放牧条件下的牧草利用 率(60\%和45\%), 因此3个刈割水平可以模拟家畜重 度放牧(C2)、中度放牧(C1) 和不放牧(C0)处理。用4 块长 $2 \mathrm{~m}$ 、宽 $0.25 \mathrm{~m}$ 雪花铁皮十字形纵切嵌入草地 $0.25 \mathrm{~m}$, 将主区样方隔成 4 个副区施肥处理样方 $(2 \mathrm{~m}$ $\times 2 \mathrm{~m})$, 其中包含 2 个施肥(F)和 2 个不施肥 $(\mathrm{NF})$ 样 方。刈割和施肥处理均在副区样方中央 $1.5 \mathrm{~m} \times 1.5$ $\mathrm{m}$ 范围内进行, 处理范围相距 $0.5 \mathrm{~m}$, 中间有铁皮相 隔, 可防止养分测向渗透。每个副区样方内再设 4 个 $0.5 \mathrm{~m} \times 0.5 \mathrm{~m}$ 的小样方, 其中 1 个为永久样方, 剩余 3 个样方用于植物性状测定(图1)。

自2007年始，每年6月上中旬进行刈割(此时间 与春季家畜放牧结束时间一致)。将刈割部分于 60 ${ }^{\circ} \mathrm{C}$ 烘干 $72 \mathrm{~h}$, 测定质量(g)。施肥于每年5、6、7月中 旬进行, 每次施尿素4.25 g.m ${ }^{-2}$ (含 $\mathrm{N} 20.4 \%$ ), $\left(\mathrm{NH}_{4}\right)_{2}$ $\mathrm{HPO}_{4} 1.02 \mathrm{~g} \cdot \mathrm{m}^{-2}$ (含 $\mathrm{N} 5.9 \%, \mathrm{P} 28.0 \%$ )。 $\mathrm{N} 、 \mathrm{P}$ 的净添 加量每年分别为 $2.782 \mathrm{~g} \cdot \mathrm{m}^{-2}$ 和 $0.857 \mathrm{~g} \cdot \mathrm{m}^{-2}$ 。本实验每 年的 $\mathrm{N}$ 素平均添加量略高于当地建植人工草地时的 中等施用量 $\left(2.25 \mathrm{~g} \cdot \mathrm{m}^{-2}\right)$, 符合当地使用 $\mathrm{N}$ 肥的实际 情况。但与在高寒草甸进行的其他施肥实验(如Yang et al., 2011)相比, 本研究的N、P添加量尚偏低。如 Yang等(2011)在甘南高寒草甸的研究, N、P的净添 加量分别在6.3-25.2 g. $\mathrm{m}^{-2}$ 和7.0-28.0 g. $\mathrm{m}^{-2}$ 之间。自 2013年开始, 我们已增加了施肥量, 其效应将在以 后的年份中逐渐显现。 


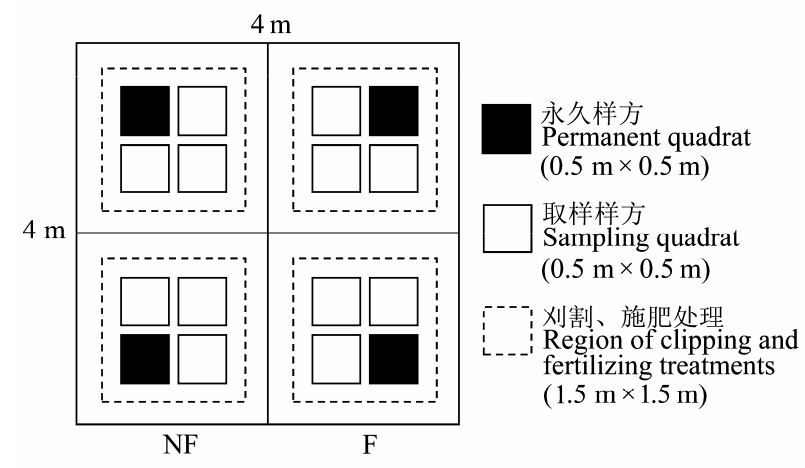

图1 副区处理样方设置图。F, 施肥; NF, 不施肥。

Fig. 1 Quadrats design layout of subplot. F, fertilized; NF, unfertilized.

\subsection{2 取样步骤与方法}

从2007年到2013年，每年8月上中旬测定永久 样方内的物种数、物种密度 (双子叶植物为个体数, 从生单子叶植物为分株数)、株高(每种植物测定 20 株, 数量少于 20 株的以实有株数测定)和总地上生 物量(2007-2008年未测), 共108个小样方。样方地上 净初级生产力以每年6月和8月两次测定的生物量之 和来估计。

本文篮选了与 $A N P P$ 显著相关的 5 个功能性状 计算功能多样性, 包括1个定量性状(株高)和 4 个定 性性状(生活周期、生长型、分类群和子叶类型)。 每种植物定性性状的分类状态根据野外观察(配合 样地外挖掘)并结合 《西藏植物志》的描述来确认, 而定量性状则采用实测值(表1)。上述植物性状能够 表征物种在刚割和施肥梯度下的耐牧或避牧、资源 利用与竞争的响应对策(Pausas et al., 2003; Canadell et al., 2007)。我们在相邻样地的研究表明, 具有某 种生活周期、生长型、分类群、子叶类型和株高性 状的物种的相对多度对家畜放牧均发生了明显响应 (Zhu et al., 2012)。在同一样地的研究显示, 不同施 肥条件下, 株高、生活周期等性状与刈割强度具有 最高的相关性(李燕和朱志红, 2013); Pillar等(2009) 在巴西的研究也发现植物生活周期类型的变化与施 氮肥和放牧扰动具有很强的相关性。

本文在计算功能多样性时首先对性状赋值进行 了标准化处理。用 0 和1表示特定植物种所属定性性 状的不存在和存在状态; 对于定量性状, 则通过对 实测值的标准化, 使物种的性状值统一映射到 $[0,1]$ 区间上进行运算(表1)。

\section{3 数据计算与统计分析}

\subsection{1 功能多样性}

功能多样性(FD)采用Rao系数(Lepš et al., 2006) 计算, 计算软件为FunctDiv (Lepš et al., 2006)。计算 公式为:

$$
F D=\sum_{i=1}^{S} \sum_{j=1}^{S} d_{i j} P_{i} P_{j}
$$

式中, $S$ 为永久样方内的物种数; $P_{i}$ 和 $P_{j}$ 分别为第 $i 、 j$ 个物种的相对多度; $d_{i j}$ 为欧氏距离, 表示物种 $i 、 j$ 在 一组性状空间中的种间性状差异。 $0 \leqslant d_{i j} \leqslant 1$, $d_{i j}=0$ 或 $d_{i j}=1$ 分别表示两物种的性状完全相同或不 同。本文计算了多性状(5个性状)的功能多样性值。

\subsection{2 统计分析}

本研究首先采用SPSS 20.0-GLM中 Repeated Measures ANOVA (RMANOVA)分析刈割和施肥处 理对 $A N P P$ 和 $F D$ 的影响, 年度为重复测定因子。通 过球形检验判断测定 $A N P P$ 和 $F D$ 在年度之间是否存 在相关性。如果球形检验中 $p<0.05$, 则使用 RMANOVA模型分析; 反之, 则使用GLM中Univariate ANOVA分析。预分析显示, 对 $A N P P$ 和 $F D$ 的球形检 验 $p$ 值分别为 0.192 和 0.306 , 满足Univariate ANOVA 的Feldt-Huynh条件。由于年份 $\times$ 刈割×施肥的交互作 用对 $A N P P$ 影响显著, 进一步采用单因素方差分析, 分年度对6种处理组合群落进行单因素方差分析, 确定其 $A N P P$ 间的差异。同理, 由于年份 $\times$ 施肥对 $F D$ 影响显著, 同样采用单因素方差分析, 对 5 个年份与 2 个施肥水平组合而成的10个处理群落进行单因素 方差分析, 确定其 $F D$ 间的差异。 $A N P P$ 和 $F D$ 在处理 间的差异采用最小显著差异法 $(L S D)$ 比较。

用SPSS 20.0-Regression程序对各处理组合群 落 $F D$ 与 $A N P P$ 在时间序列上的关系进行对数尺度回 归分析, 以 $F$ 检验显著确定线性回归模型。最后, 将 一种处理因素控制在单一水平(如不施肥), 以另一 种处理因素作为固定因子 (如则割强度), 以 $F D$ 作为 协变量进行协方差分析(ANCOVA), 检验施肥或刈 割水平对FD-ANPP回归关系的影响。若协变量与某 一处理因素的交互作用显著, 则该处理因素对回归 斜率有显著性影响; 若回归截距项检验显著, 则该 处理因素仅对ANPP有显著影响。统计分析前, 对数 据进行对数转换, 以满足ANOVA中正态性和方差 同质性的假定。以上统计分析的显著性水平均设为 $p<0.05$ 。使用Origin 8.5软件作图。由于2007-2008 
表1 计算功能多样性指数的功能性状及其分类状态

Table 1 Plant functional traits and their categories for calculating functional diversity

\begin{tabular}{lll}
\hline 性状类型 Trait types & 性状名称 Trait names & 性状状态性状值 Trait states/Trait value \\
\hline 定性性状 Qualitative trait & 生活周期 Life cycle & 非多年生 Not perennial; 多年生 Perennial \\
& 生长型 Growth form & 散生 Scattered; 丛生 Bunched; 密从生 Closely bunched \\
& 分类群 Taxonomic group & 禾草 Gramineae; 莎草 Cyperaceae; 豆科植物 Leguminosae; 杂类草 Forbs \\
& 子叶类型 Cotyledon type & 单子叶 Monocotyledon; 双子叶 Dicotyledon \\
定量性状 Quantitative trait & 株高 Plant height & 标准化实测值 Standardization of measured values \\
\hline
\end{tabular}

表2２009-2013年施肥、刈割对高寒草甸地上净初级生产力和功能多样性影响的方差分析

Table 2 ANOVA for the effects of clipping, fertilizing on the aboveground net primary productivity and functional diversity in alpine meadow during 20092013

\begin{tabular}{|c|c|c|c|c|c|c|}
\hline \multirow{2}{*}{\multicolumn{2}{|c|}{$\begin{array}{l}\text { 变异来源 } \\
\text { Source of variance }\end{array}$}} & \multirow{3}{*}{$\begin{array}{c}\begin{array}{c}\text { 自由度 } \\
d f(m, n)\end{array} \\
2,28\end{array}$} & \multicolumn{2}{|c|}{ ANPP } & \multicolumn{2}{|c|}{$F D$} \\
\hline & & & \multirow{2}{*}{$\frac{F \text { 检验 } F \text {-test }}{1.09}$} & \multirow{2}{*}{$\begin{array}{c}p \\
0.349\end{array}$} & \multirow{2}{*}{$\frac{F \text { 检验 } F \text {-test }}{1.780}$} & \multirow{2}{*}{$\begin{array}{r}p \\
0.187\end{array}$} \\
\hline 主区 & B & & & & & \\
\hline \multirow[t]{3}{*}{ Whole plot } & C & 2,28 & 4.04 & $0.029^{* * *}$ & 1.840 & $0.178_{* *}$ \\
\hline & $\mathrm{Y}$ & 4,28 & 82.90 & $0.000^{* *}$ & 41.450 & $0.000^{* *}$ \\
\hline & $\mathrm{Y} \times \mathrm{C}$ & 8,28 & 15.41 & $0.000^{* *}$ & 0.690 & 0.697 \\
\hline 副区 & $\mathrm{F}$ & 1,30 & 375.75 & $0.000^{* *}$ & 36.720 & $0.000^{* *}$ \\
\hline \multirow{4}{*}{ Subplot } & $\mathrm{C} \times \mathrm{F}$ & 2,30 & 2.37 & 0.111 & 1.870 & 0.172 \\
\hline & $\mathrm{Y} \times \mathrm{F}$ & 4,30 & 6.46 & $0.001^{* *}$ & 3.190 & $0.027^{*}$ \\
\hline & $\mathrm{Y} \times \mathrm{C} \times \mathrm{F}$ & 8,30 & 3.18 & $0.010^{*}$ & 0.470 & 0.869 \\
\hline & $\mathrm{Y} \times \mathrm{C} \times \mathrm{B}$ & 28,30 & 1.58 & 0.110 & 2.361 & $0.011^{*}$ \\
\hline
\end{tabular}

*, $p<0.05 ; * *, p<0.01$ 。 , 处理自由度; $n$, 误差自由度。 $\mathrm{B}$, 区组; $\mathrm{C}$, 刈割; $\mathrm{Y}$, 年份; $\mathrm{F}$, 施肥; $A N P P$, 地上净初级生产力; $F D$, 功能多样性。 $\times$, 交互 作用。

${ }^{*}, p<0.05 ; * *, p<0.01 . m$, degree of freedom in treatment; $n$, degree of freedom in error. B, block; C, clipping; $\mathrm{Y}$, year; $\mathrm{F}$, fertilizing; ANPP, aboveground net primary productivity; $F D$, functional diversity. $\times$, interaction.

年未获得 $A N P P$ 数据, 用于本研究分析的数据均采 自2009-2013年。

\section{2 结果分析}

\section{1 刏割和施肥对 $A N P P$ 的影响}

刈割、年份、施肥因子, 以及年份 $\times$ 刏割、年份 $\times$ 施肥、年份 $\times$ 刈割 $\times$ 施肥的交互作用对 $A N P P$ 影响显 著，其他因子及交互作用均不显著(表2)。随着刚割 强度增加以及施肥, $A N P P$ 显著增加。ANPP在年度间 总体上呈现波动性下降趋势(图2), 且受施肥和刈割 的影响。不施肥时, ANPP在实验前期(2009-2011年) 因刚割显著降低(2009年，2011年)或无显著变化 (2010年), 在后期(2012-2013年)则随刈割强度增加 而显著提高(图3)。与不施肥相比, 施肥增加了各年 度刈割处理的 $A N P P$, 但并未在根本上改变ANPP在 刈割处理间以及年度间的变化格局(图3)。

\section{2 刏割和施肥对 $F D$ 的影响}

年份、施肥、年份 $\times$ 施肥、年份 $\times$ 刈割 $\times$ 区组对 $F D$ 影响显著, 其他因子及交互作用均不显著(表2)。 $F D$ 在施肥后显著提高, 并随时间增加, 特别是在 2009-2010年间增加程度较大(图4)。由于刈割和年 份 $\times$ 刈割对 $F D$ 无影响, $F D$ 随年度增加应主要是受施
肥的影响(表2)。与不施肥相比, 施肥后 $F D$ 在实验前 期(2009-2011)显著增加，在后期(2012-2013)则无 显著变化(图5)。

\section{3 施肥和划割对FD-ANPP关系的影响}

在2009-2013年时间序列上, FD-ANPP关系表 现为无相关和正相关两种形式(图6), 即无论施肥与 否, 不刚割群落的 $F D$ 与 $A N P P$ 均无相关性(不划割不 施肥: $F_{\mathrm{CONF}}=0.926, R^{2}=0.010, n=90, p=0.338$; 不 刈割施肥: $F_{\mathrm{COF}}=0.949, R^{2}=0.011, n=90, p=$ $0.333)$, 而刈割的 4 种群落均为正线性相关, 其 $F D(x)$ 与ANPP $(y)$ 的关系方程分别为: 中度刈割不施肥: $y_{\mathrm{C} 1 \mathrm{NF}}=0.54 x+2.13\left(F=9.832, R^{2}=0.101, n=90, p\right.$ $=0.002)$; 中度刈割施肥: $y_{\mathrm{C} 1 \mathrm{~F}}=0.55 x+2.28(F=$ 4.903, $\left.R^{2}=0.053, n=90, p=0.029\right)$; 重度刈割不施 肥: $y_{\mathrm{C} 2 \mathrm{NF}}=1.09 x+1.78\left(F=19.049, R^{2}=0.178, n=\right.$ $90, p=0.000)$; 重度刚割施肥: $y_{\mathrm{C} 2 \mathrm{~F}}=0.66 x+2.22(F$ $\left.=5.955, R^{2}=0.063, n=90, p=0.017\right)$ 。在C2NF群落 中, 方程的斜率为 $1.091, A N P P$ 的增加速度大于 $F D$; 在其他群落, 斜率均小于 $1, A N P P$ 的增加速度小于 $F D$ 。

协方差分析的结果显示: 在不施肥(NF)群落, 刏割显著增加了 $F D-A N P P$ 关系的斜率 $\left(F_{(2,264)}=\right.$ 

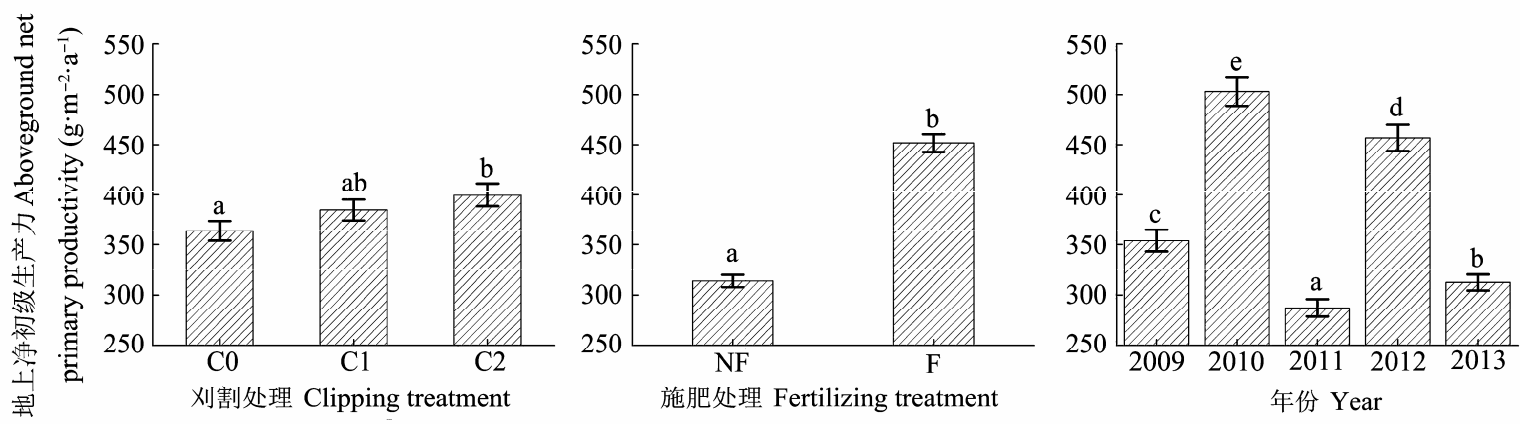

图2 刈割、施肥和年份对地上净初级生产力的影响(平均值土标准误差)。C0、C1、C2、F和NF分别表示不刚割、中度刈割、 重度刈割、施肥和不施肥处理。标准误差上方相同的字母表示处理间差异不显著 $(p>0.05)$, 不同字母表示处理间差异显著 $(p<$ 0.05)。

Fig. 2 Effects of clipping, fertilizing and year on the aboveground net primary productivity (mean $\pm S E$ ). C0, C1, C2, F and NF indicate no clipping, moderate clipping, heavy clipping, fertilizing and no fertilizing. The same letter above error bars indicates no difference among treatments $(p>0.05)$, and different letters indicate significant differences among treatments $(p<0.05)$.
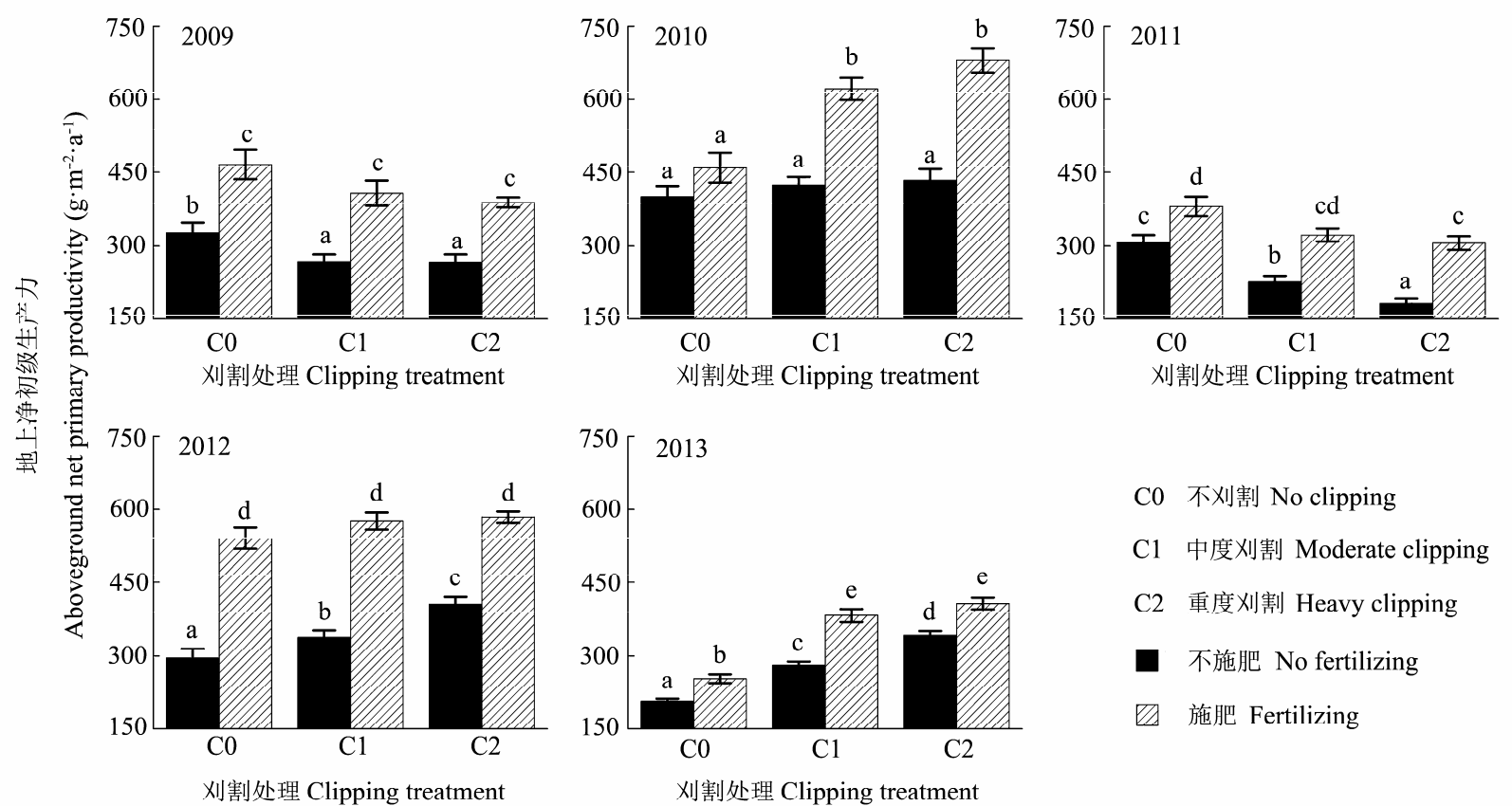

图3 2009-2013年刈割和施肥对地上净初级生产力的影响(平均值土标准误差)。标准误差上方相同的字母表示处理间差异不显 著 $(p>0.05)$, 不同字母表示处理间差异显著 $(p<0.05)$ 。

Fig. 3 Effects of clipping and fertilizing on aboveground net primary productivity during 2009-2013 (mean $\pm S E$ ). The same letter above error bars indicates no difference among treatments $(p>0.05)$, and different letters indicate significant differences between treatments $(p<0.05)$.

9.085, $p<0.001)$, 而减小了截距 $\left(F_{(1,264)}=84668.477\right.$, $p<0.001)$; 在施肥 $(\mathrm{F})$ 群落, 刈割同样增加了斜率 $\left(F_{(2,264)}=3.433, p=0.034\right)$, 减小了截距 $\left(F_{(1,264)}=\right.$ 95 056.370, $p<0.001$ ); 在中度刚割(C1)群落, 施肥 对斜率影响不显著 $\left(F_{(1,176)}=0.001, p=0.977\right)$, 但增 加了截距 $\left(F_{(1,176)}=66760.585, p<0.001\right)$; 在重度 刏割(C2)群落, 施肥后斜率也无显著变化 $\left(\mathrm{F}_{(1,176)}=\right.$ 1.343, $p=0.248)$, 而截距增加 $\left(\mathrm{F}_{(1,176)}=57280.001\right.$, $p<0.001)$ 。上述结果说明, 刈割与否是决定 $F D-A N P P$ 关系性质的首要因素, 因为不刈割时为无 相关, 且与施肥无关, 而刈割后均为正相关。但刈割 后正相关的特点受刚割强度和施肥与否的影响。也 就是说, 刈割强度越大的群落ANPP随FD的变化速 率越高, $F D$ 对 $A N P P$ 的影响越强, 而施肥并不影响 $F D$ 对 $A N P P$ 的作用强度, 仅是同步提高了不同 $F D$ 水 平的 $A N P P$ 。 

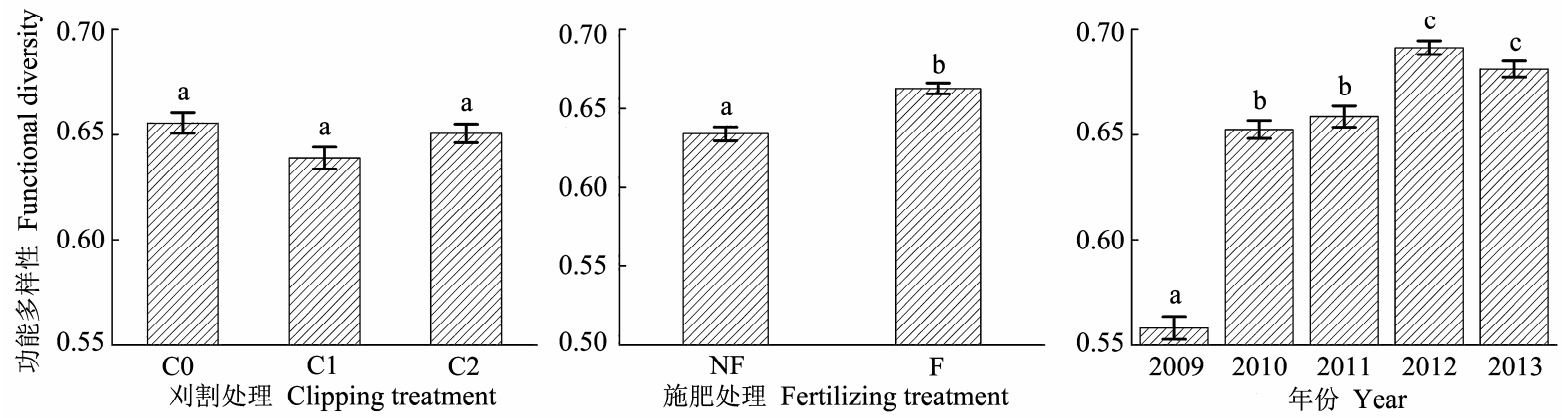

图4 刈割、施肥和年份对功能多样性的影响(平均值标准误差)。C0、C1、C2、F和NF分别表示不刈割、中度刈割、重度刈 割、施肥和不施肥处理。标准误差上方相同的字母表示处理间差异不显著 $(p>0.05)$, 不同字母表示处理间差异显著 $(p<0.05)$ 。 Fig. 4 Effects of clipping, fertilizing and year on the functional diversity (mean $\pm S E$ ). C0, C1, C2, F and NF indicate no clipping, moderate clipping, heavy clipping, fertilizing and no fertilizing. The same letter above error bars indicates no difference among treatments, and different letters indicate significant differences between treatments $(p<0.05)$.

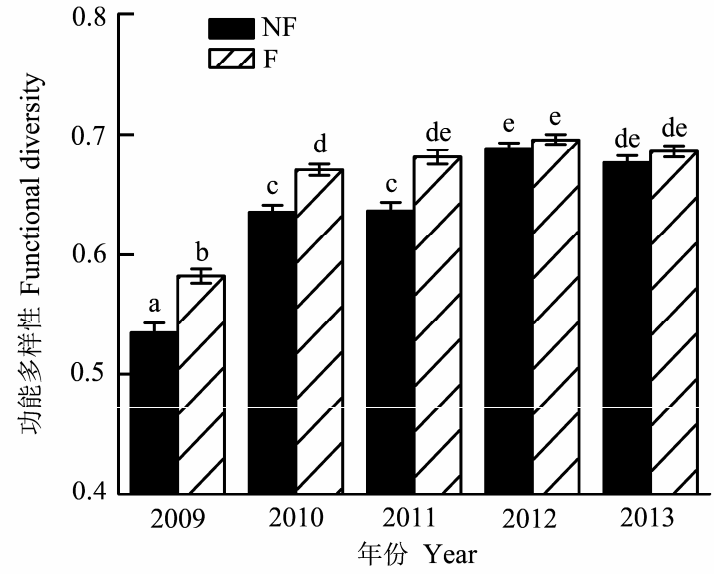

图5 2009-2013年施肥对功能多样性的影响(平均值土标准 误差)。 $\mathrm{F}$, 施肥; NF, 不施肥。不同字母表示处理间差异显著 $(p<0.05)$ 。

Fig. 5 Effects of fertilizing on functional diversity during 2009-2013 (mean $\pm S E$ ). F, fertilizing; NF, no fertilizing. Different letters indicate significant differences between treatments $(p<0.05)$.

\section{3 讨论}

\section{1 刏割与施肥对ANPP和FD的影响}

本研究表明, 增加刈割强度能提高ANPP (图

2)。这种作用应该与刚割引起植物的超补偿生长(刘 建军等, 2005)有关。周晓松等(2011)在同一样地的研 究证实: 尽管刈割抑制了高大禾草的生长, 而莎草 科植物以及一些阔叶类杂草的超补偿生长是导致群 落ANPP增加的重要原因。施肥增加ANPP (图2)与很 多研究结果(高本强等, 2014; Niu et al., 2014; 杨晓 霞等, 2014)相同, 因为施肥后高大的禾本科植物垂 穗披碱草、莎草科植物矮生嵩草、阔叶双子叶植物
美丽风毛菊等很多植物种的重要值显著增加, 提高 了群落初级生产力(周晓松等, 2011)。这也直观反映 了受低温控制的高寒草甸土壤速效养分缺乏的实际, 而施肥则能有效地解除这一限制作用。

刚割强度处理间FD无显著差异(图4), 可能与 Rao系数中包含的种间性状差异 $\left(d_{i j}\right)$ 和物种均匀度 $\left(P_{i} P_{j}\right)$ 这两个成分对刈割的不同响应有关。陈超等 (2015)在本样地的研究表明: 随着刈割强度增加, 种间性状差异和物种均匀度分别表现为减小和增加 的反向变化, 导致 $F D$ 在刚割处理间无显著变化。

很多研究认为施肥后 $F D$ 会随着物种的丧失而 减小(Tilman \& Downing, 1994; Hooper et al., 2005; Moonen \& Bàrberi, 2008), 我们利用采自2012年13 个植物功能性状数据的研究也得到相同结果(陈超 等, 2015)。但本文结果却显示施肥后 $F D$ 的时间平均 值(2009-2013)显著增加(图4)。Niu等(2014)认为施肥 引起 $F D$ 的变化与两个相反的机制有关: 一是竞争 排斥会导致FD减小(Leibold, 1998; Grime, 2006; Mayfield \& Levine, 2010); 二是共存物种间的生态 位分化又会促使性状趋异, 引起FD增加(Macarthur \& Levins, 1967; Navas \& Violle, 2009; Mouchet et al., 2010; Mason et al., 2011), FD对施肥的响应取决于 这两种机制的平衡(Niu et al., 2014)。本文结果可能 与第二种机制有关, 即共存物种生态位分化对 FD 的影响超过了物种丧失的作用。原因有以下 2 个: (1) 在本研究样地不施肥和施肥群落共有物种的比例仍 高达93\%和94\%, 施肥降低物种多样性仅发生在不 刈割处理, 而刈割处理的多样性并未下降(王海东 

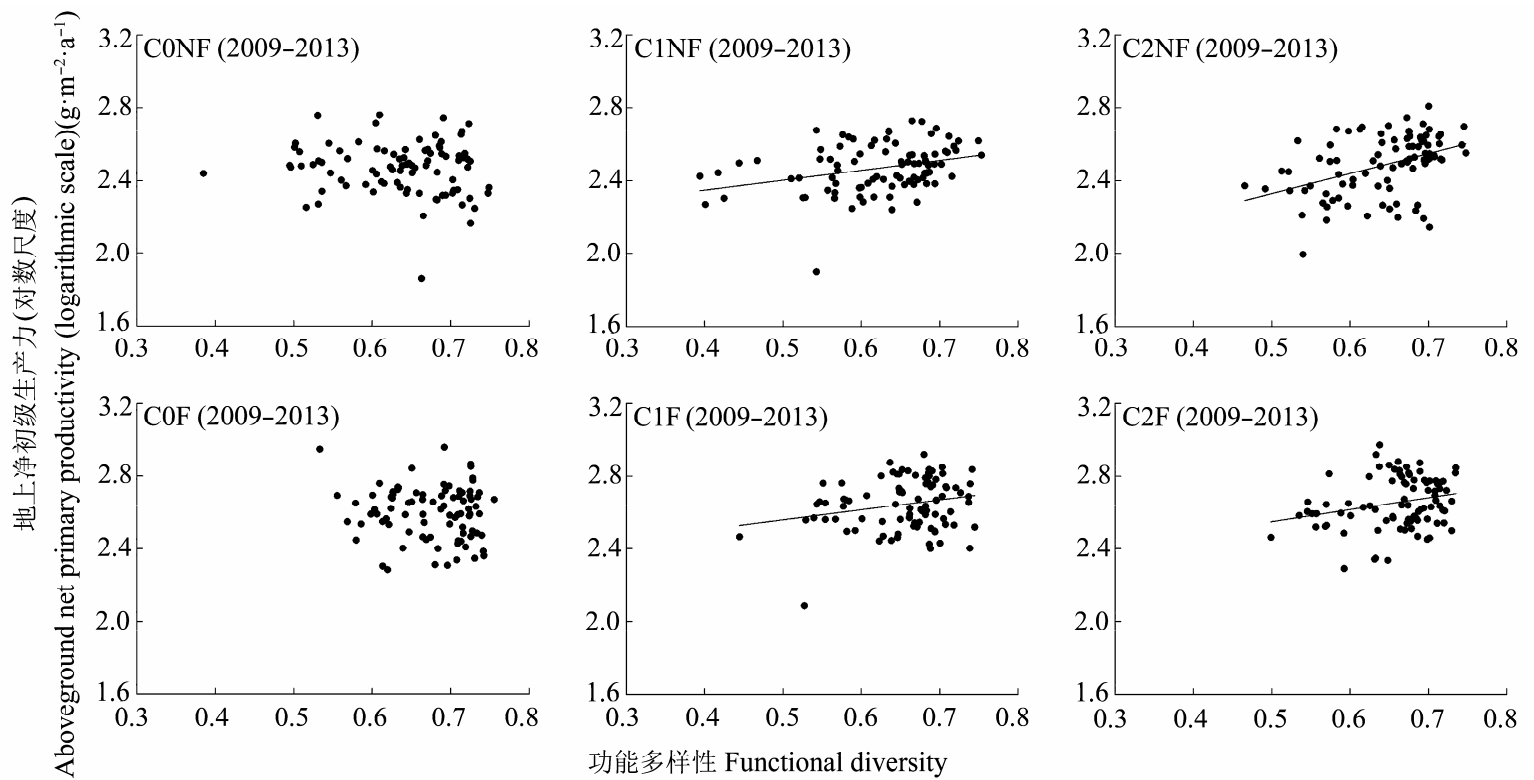

图6 2009-2013年刚割和施肥组合梯度中功能多样性和地上净初级生产力的关系(对数尺度) $(p<0.05) 。 \mathrm{C} 0 、 \mathrm{C} 1 、 \mathrm{C} 2 、 \mathrm{~F} 、 \mathrm{NF}$ 同图2。

Fig. 6 Relationships between functional diversity and aboveground net primary productivity (logarithmic scale) in different gradients of clipping and fertilizing treatments $(p<0.05)$. C0, C1, C2, F, NF see Fig. 2.

等, 2013)。由于物种的周转率仅为 $6 \%-7 \%$, 且多为 稀有种, 因此, 尽管施肥显著降低了物种多样性和 物种均匀度, 但尚不足以导致 $F D$ 显著减小。(2)在本 样地及与之相邻的家畜放牧样地的研究显示, 在不 刈割+施肥处理或富养草地群落中, 垂穗披碱草和 异针茅等禾本科植物的优势度都很高, 而阔叶杂类 草的优势度则很低(藏岳铭等, 2009; 周晓松等, 2011)。说明施肥在解除土壤养分限制作用的同时也 增强了种间的光竞争, 施肥后引起物种在光资源利 用上的不对称性竞争导致种间生态位分化, 施肥群 落 $F D$ 的时间平均值较高。

\section{$3.2 \quad A N P P$ 和F $D$ 的年度动态}

$A N P P$ 随时间呈现波动性下降的趋势(图2), 而 施肥并未在根本上改变刈割处理间ANPP随年度变 化的格局(图3), 因此, ANPP的波动性下降极有可能 与年度间气候条件的变化有关。在干旱草原生态系 统的很多研究也表明降水量与 $A N P P$ 的波动具有极 高的同步性(Isbell et al., 2009)。然而, 我们使用定位 站地区2009-2013年年总降水量、生长季降水量、年 平均气温、生长季平均气温和草地干燥度(即生长季 积温/生长季降水量)与ANPP进行回归分析, 发现相 关性并不显著 $(p$ 值分别为 $0.594 、 0.692 、 0.733 、 0.683$ 和0.613), 而且这些气候指标与 $A N P P$ 的年度波动趋
势也不一致, 说明 $A N P P$ 的波动性下降与气候变化 并无直接关系。同一样地的研究显示, 长期刈割会 显著降低植物的再生高度(李晓刚等, 2011), 植物趋 于矮化以适应刈割扰动。我们推测本研究中 $A N P P$ 的波动性下降可能与株高的变化有关。但回归分析 显示二者并无相关性 $(p=0.744)$ 。另有研究显示, 长 期刚割后植物根系陉藏的碳水化合物的大量消耗会 降低植物的补偿再生能力, 引起ANPP逐年降低(许 志信等, 1993)。但由于本研究不刈割处理的ANPP 也同样表现出逐年下降的趋势(图3), 因此, 根系咜 藏碳水化合物的变化也不能对此给出满意的解释。 本研究中 ANPP随年度波动性下降的原因还有待进 一步研究。

本研究连续7年的刚割和施肥处理已经引起群 落出现明显的异质性, 不同处理控制下的实验草地 正向不同的群落状态发展。尽管刈割对 $F D$ 未产生显 著影响, 但施肥却提高了FD (图4), 反映了群落总 体在种间性状差异和物种均匀度等方面发生分异的 时间过程, 即人为扰动形成的群落空间异质性导致 群落总体的功能多样性随时间增大。此结果与空间 异质性提高群落物种多样性的效应(Hooper et al., 2005)相似。可以预计, 随着时间的延续, 群落总体 $F D$ 将进一步变化, 直至扰动因素与种内、种间相互 
作用达到平衡为止。

\section{$3.3 F D-A N P P$ 的关系模式}

与很多研究不同的是, 本研究中 FD-ANPP的时 间相关性并未出现多种模式(Thompson et al., 2005; Griffin et al., 2009; 藏岳铭等, 2009; 吕亭亭等, 2014), 而是表现为不刚割群落的无相关及刈割群 落的正线性相关两种模式, 而且相关模式的变化强 度依赖于刈割强度, 与施肥无关(图6), 进一步证实 了 FD-ANPP关系与扰动因素存在密切联系的观点 (Bernhardt-Römermann et al., 2011; 李晓刚等, 2011)。

需要强调的是, 本研究主要关注 $F D-A N P P$ 的时 间相关性, 以期探讨自然群落在遭受某种环境外力 影响后群落物种功能多样性与生产力关系的长期变 化。本研究中的不刚割群落相当于封育恢复中的植 物群落, 由于7年未受扰动, 群落已完全恢复到以高 大的禾本科植物为优势种(周晓松等, 2011), 并对下 层植物形成了稳固的光竞争格局, $F D$ 的分异减小, 并与ANPP无显著相关性(图6)。而在扰动条件下, 若 扰动对多样性和生产力同时产生正效应, 就可能出 现多样性与生产力的正相关关系(Loreau, 2000)。同 理, 由于 $F D$ 和 $A N P P$ 对刈割扰动都呈正反应, 因此 $F D-A N P P$ 关系表现为正相关(图6)。从生态互补的观 点来看, 刏割扰动会释放一定的竞争空间, 植物能 更加有效地利用各种资源。随着刈割强度增加, 释 放的空间越大, 低矮植物逐渐成为优势种, 在相同 的 $F D$ 条件下, ANPP增幅也更大。因此, 刏割强度越 大的群落, ANPP随FD的变化速率越高, FD对ANPP 的影响越强(图6)。

综上所述, 刈割扰动和土壤资源状况共同影响 着FD-ANPP的关系, 其中刈割扰动在决定二者关系 性质时起主导作用。我们推测在放牧状态下的高寒 草甸群落, 由多性状计算的功能多样性能较好地预 测群落生产力的变化。而不刚割群落功能多样性的 逐年下降及其生产力低于刚割群落, 表明长期禁牧 并不利于高寒草甸的管理, 因此适当放牧并增加土 壤可利用资源可以使多样性和生产力保持在较高 水平。

\section{基金项目 国家自然科学基金(31570425)。}

致谢 感谢中国科学院海北高寒草甸生态系统定位 研究站的支持, 同时也感谢陕西师范大学生态学研 究室吕美强、陈超、李艳、关百盈、杜家丽等同学
在野外数据采集过程中的辛苦付出。

\section{参考文献}

Bernhardt-Römermann M, Römermann C, Sperlich S, Schmidt W (2011). Explaining grassland biomass-The contribution of climate, species and functional diversity depends on fertilization and mowing frequency. Journal of Applied Ecology, 48, 1088-1097.

Canadell JG, Pataki DE, Pitelka LF (2007). Terrestrial Ecosystems in a Changing World. Springer-Verlag, Berlin.

Chapin FS III, Zavaleta ES, Eviner VT, Naylor RL, Vitousek PM, Reynolds HL, Hooper DU, Lavorel S, Sala OE, Hobbie SE, Mack MC, Díaz S (2000). Consequences of changing biodiversity. Nature, 405, 234-242.

Chen C, Zhu ZH, Li YN, Yao TH, Pan SY, Wei XH, Kong BB, Du JL (2015). Effects of trait dissimilarity among species and species evenness on the relationship between species diversity and functional diversity in alpine meadow. Acta Ecologica Sinica, DOI: 10. 5846/stxb 201405070903. (in Chinese with English abstract) (in Press) [陈超, 朱志红, 李英年, 姚天华, 潘石玉, 卫欣华, 孔涁涁, 杜家丽 (2015). 高寒草甸种间性状差异和物种均匀度对物种多 样性与功能多样性关系的影响. 生态学报, DOI: 10 . 5846/stxb201405070903.](待发表)

Díaz S, Cabido M (2001). Vive la différence: Plant functional diversity matters to ecosystem processes. Trends in Ecology \& Evolution, 16, 646-655.

Gao BQ, Yuan ZQ, Wang BX, Gao H, Zhang R (2014). Effects of fertilization and clipping on species diversity, productivity and their relationship in subalpine meadow. Chinese Journal of Plant Ecology, 38, 417-424. (in Chinese with English abstract) [高本强, 袁自强, 王斌先, 高慧, 张荣 (2014). 施肥和刚割对亚高山草甸物种多样性与生产力 及其关系的影响. 植物生态学报, 38, 417-424.]

Griffin JN, Méndez V, Johnson AF, Jenkins SR, Foggo A (2009). Functional diversity predicts overyielding effect of species combination on primary productivity. Oikos, 118, $37-44$.

Grime JP (2006). Trait convergence and trait divergence in herbaceous plant communities: Mechanisms and consequences. Journal of Vegetation Science, 17, 255-260.

Hillebrand H, Matthiessen B (2009). Biodiversity in a complex world: Consolidation and progress in functional biodiversity research. Ecology Letters, 12, 1405-1419.

Hooper DU (1998). The role of complementarity and competition in ecosystem responses to variation in plant diversity. Ecology, 79, 704-719.

Hooper DU, Chapin FS III, Ewel JJ, Hector A, Inchausti P, Lavorel S, Lawton JH, Lodge DM, Loreau M, Naeem S, Schmid B, Setälä H, Symstad AJ, Vandermeer J, Wardle DA (2005). Effects of biodiversity on ecosystem functioning: A consensus of current knowledge. Ecological 
Monographs, 75, 3-35.

Isbell FI, Polley HW, Wilsey BJ (2009). Biodiversity, productivity and the temporal stability of productivity: Patterns and processes. Ecology Letters, 12, 443-451.

Jiang XL, Zhang WG (2010). Functional diversity and its research method. Acta Ecologica Sinica, 30, 2766-2773. (in Chinese with English abstract) [江小雷, 张卫国 (2010). 功能多样性及其研究方法. 生态学报, 30, 2766-2773.]

Kaiser J (2000). Rift over biodiversity divides ecologists. Science, 289, 1282-1283.

Leibold MA (1998). Similarity and local co-existence of species in regional biotas. Evolutionary Ecology, 12, 95-110.

Lepš J, Brown VK, Diaz Len TA, Gormsen D, Hedlund K, Kailová J, Korthals GW, Mortimer SR, RodriguezBarrueco C, Roy J, Regina IS, Van Dijk C, van der Putten WH (2001). Separating the chance effect from other diversity effects in the functioning of plant communities. Oikos, 92, 123-134.

Lepš J, de Bello F, Lavorel S, Berman S (2006). Quantifying and interpreting functional diversity of natural communities: Practical considerations matter. Preslia, 78, 481-501.

Li XG, Zhu ZH, Zhou XS, Yuan FR, Fan RJ, Xu ML (2011). Effects of clipping, fertilizing and watering on the relationship between species diversity, functional diversity and primary productivity in alpine meadow of China. Chinese Journal of Plant Ecology, 35, 1136-1147. (in Chinese with English abstract) [李晓刚, 朱志红, 周晓松, 袁芙蓉, 訤 瑞俭, 许曼丽 (2011). 刈割、施肥和浇水对高寒草甸物 种多样性、功能多样性与初级生产力关系的影响. 植物 生态学报, 35, 1136-1147.]

Li Y, Zhu ZH (2013). Optimal plant traits and plant functional types responsible to clipping, fertilizing and watering in alpine meadow. Chinese Journal of Plant Ecology, 37, 384-396. (in Chinese with English abstract) [李燕, 朱志 红 (2013). 高寒草甸对刈割、施肥和浇水发生响应的最 优植物性状集和功能型. 植物生态学报, 37, 384-396.]

Li YN, Wang QX, Gu S, Fu YL, Du MY, Zhao L, Zhao XQ, Yu GR (2004). Integrated monitoring of alpine vegetation types and its primary production. Acta Geographica Sinica, 59, 40-48. (in Chinese with English abstract) [李英 年, 王勤学, 古松, 伏玉玲, 杜明远, 赵亮, 赵新全, 于 贵瑞 (2004). 高寒植被类型及其植物生产力的监测. 地 理学报, 59, 40-48.]

Liu JJ, Urano T, Mariko S, Mariko S, Oikawa T (2005). Influence of grazing pressures on belowground productivity and biomass in Mongolia steppe. Acta Botanica BorealiOccidentalia Sinica, 25, 88-93. (in Chinese with English abstract) [刘建军, 浦野忠朗, 鞠子茂, 及川武久 (2005). 放牧对草原生态系统地下生产力及生物量的影 响. 西北植物学报, 25, 88-93.]
Loreau M (2000). Biodiversity and ecosystem functioning: Recent theoretical advances. Oikos, 91, 3-17.

Lü TT, Wang P, Yan H, Zhang W, Liao GD, Jiang HB, Zou CL, Sheng LX (2014). Relationship between functional diversity and productivity in meadow and marsh plant communities. Chinese Journal of Plant Ecology, 38, 405416. (in Chinese with English abstract) [吕亭亭, 王平, 燕 红, 张稳, 廖桂项, 姜海波, 邹畅林, 盛连喜 (2014). 草 甸和沼泽植物群落功能多样性与生产力的关系. 植物 生态学报, 38, 405-416.]

Macarthur R, Levins R (1967). The limiting similarity, convergence, and divergence of coexisting species. The American Naturalist, 101, 377-385.

Mason NWH, de Bello F, Doležal J, Lepš J (2011). Niche overlap reveals the effects of competition, disturbance and contrasting assembly processes in experimental grassland communities. Journal of Ecology, 99, 788-796.

Mayfield MM, Levine JM (2010). Opposing effects of competitive exclusion on the phylogenetic structure of communities. Ecology Letters, 13, 1085-1093.

Mokany K, Ash J, Roxburgh S (2008). Functional identity is more important than diversity in influencing ecosystem processes in a temperate native grassland. Journal of Ecology, 96, 884-893.

Moonen AC, Bàrberi P (2008). Functional biodiversity: An agroecosystem approach. Agriculture, Ecosystems \& Environment, 127, 7-21.

Mouchet MA, Villéger S, Mason NW, Mouillot D (2010). Functional diversity measures: An overview of their redundancy and their ability to discriminate community assembly rules. Functional Ecology, 24, 867-876.

Naeem S (2002). Ecosystem consequences of biodiversity loss: The evolution of a paradigm. Ecology, 83, 1537-1552.

Navas ML, Violle C (2009). Plant traits related to competition: How do they shape the functional diversity of communities? Community Ecology, 10, 131-137.

Niu KC, Choler P, de Bello F, Mirotchnick N, Du GZ, Sun SC (2014). Fertilization decreases species diversity but increases functional diversity: A three-year experiment in a Tibetan alpine meadow. Agriculture, Ecosystems \& Environment, 182, 106-112.

Pausas JG, Carreras J, Ferré A, Font X (2003). Coarse-scale plant species richness in relation to environmental heterogeneity. Journal of Vegetation Science, 14, 661-668.

Petchey OL, Gaston KJ (2002). Functional diversity (FD), species richness and community composition. Ecology Letters, 5, 402-411.

Petchey OL, Gaston KJ (2006). Functional diversity: Back to basics and looking forward. Ecology Letters, 9, 741-758.

Pillar VD, Duarte LDS, Sosinski EE, Joner F (2009). Discriminating trait-convergence and trait-divergence assembly patterns in ecological community gradients. Journal of 
Vegetation Science, 20, 334-348.

Schleuter D, Daufresne M, Massol F, Argillier C (2010). A user's guide to functional diversity indices. Ecological Monographs, 80, 469-484.

Thompson K, Askew AP, Grime JP, Dunnett NP, Willis AJ (2005). Biodiversity, ecosystem function and plant traits in mature and immature plant communities. Functional Ecology, 19, 355-358.

Tilman D, Downing JA (1994). Biodiversity and stability in grasslands. Nature, 367, 363-365.

Tilman D, Knops J, Wedin D, Reich P, Ritchie M, Siemann E (1997). The influence of functional diversity and composition on ecosystem processes. Science, 277, 1300-1302.

Tilman D, Reich PB, Knops J, Wedin D, Mielke T, Lehman C (2001). Diversity and productivity in a long-term grassland experiment. Science, 294, 843-845.

Tilman D, Wedin D, Knops J (1996). Productivity and sustainability influenced by biodiversity in grassland ecosystems. Nature, 379, 718-720.

Wang CT, Long RJ, Wang QJ, Jing ZC, Ding LM (2005). Relationship between species diversity and productivity in four types of alpine meadow plant communities. Chinese Journal of Ecology, 24, 483-487. (in Chinese with English abstract) [王长庭, 龙瑞军, 王启基, 景增春, 丁路明 (2005). 高寒草甸不同草地群落物种多样性与生产力关 系研究. 生态学杂志, 24, 483-487.]

Wang HD, Zhang LL, Zhu ZH (2013). Effects of clipping and fertilizing on the relationships between species diversity and ecosystem functioning and mechanisms of community stability in alpine meadow. Chinese Journal of Plant Ecology, 37, 279-295. (in Chinese with English abstract) [王海东, 张璐璐, 朱志红 (2013). 刈割、施肥对高寒草 甸物种多样性与生态系统功能关系的影响及群落稳定 性机制. 植物生态学报, 37, 279-295.]

Xu ZX, Batu CL, Wei ZJ, Duan CQ, Zhao G, Zhaohe ST (1993). Relationship between herbage regrowth and dynamics of carbohydrate storage. Acta Prataculturae Sinica, 2(4), 13-18. (in Chinese with English abstract) [许 志信, 巴图朝鲁, 卫智军, 段淳清, 赵刚, 昭和斯图 (1993). 牧草再生与捾藏碳水化合物含量变化关系的研 究. 草业学报, 2(4), 13-18.]

Yang XX, Ren F, Zhou HK, HE JS (2014). Responses of plant community biomass to nitrogen and phosphorus additions in an alpine meadow on the Qinghai-Xizang Plateau. Chinese Journal of Plant Ecology, 38, 159-166. (in Chinese with English abstract) [杨晓霞, 任飞, 周华坤, 贺金生 (2014). 青藏高原高寒草甸植物群落生物量对氮、磷添 加的响应. 植物生态学报, 38, 159-166.]

Yang ZL, van Ruijven J, Du GZ (2011). The effects of long-term fertilization on the temporal stability of alpine meadow communities. Plant and Soil, 345, 315-324.

Zang YM, Zhu ZH, Li YN, Wang WJ, Xi B (2009). Effects of species diversity and functional diversity on primary productivity of alpine meadow. Chinese Journal of Ecology, 28, 999-1005. (in Chinese with English abstract) [㶓岳铭, 朱志红, 李英年, 王文娟, 席博 (2009). 高寒矮嵩草草 甸物种多样性与功能多样性对初级生产力的影响. 生 态学杂志, 28, 999-1005.]

Zhao XQ (2009). Global Change and Ecological System in Alpine Meadow. Science Press, Beijing. 78. (in Chinese) [赵新全 (2009). 高寒草甸生态系统与全球变化. 科学 出版社, 北京. 78.]

Zhou XS, Zhu ZH, Li YN, Yuan FR, Fan RJ (2011). Community compensatory mechanism under clipping, fertilizing and watering treatment in alpine meadow. Journal of Lanzhou University (Natural Sciences), 47(3), 50-57. (in Chinese with English abstract) [周晓松, 朱志红, 李英年, 袁芙蓉, 採瑞俭 (2011). 刈割、施肥和浇水处理下高寒 矮高草草甸补偿机制. 兰州大学学报 (自然科学版), 47(3), 50-57.]

Zhu ZH, Wang G (1996). An approach to analyzing nature of community structure: With examples of alpine meadow and alpine bushland. Acta Phytoecologica Sinica, 20, 184-192. (in Chinese with English abstract) [朱志红, 王 刚 (1996). 群落结构特性的分析方法探讨一以高寒 草甸和高寒灌丛为例. 植物生态学报, 20, 184-192.]

Zhu ZH, Wang G, Zhao SL (1994). Dynamics and regulation of clonal ramet population in Komresia humilis under different stocking intensities. Acta Ecologica Sinica, 14, 40-45. (in Chinese with English abstract) [ 朱志红, 王刚, 赵松龄 (1994). 不同放牧强度下矮嵩草(Kobresia humilis)无性 系分株种群的动态与调节. 生态学报, 14, 40-45.]

Zhu ZH, Wang XA, Li YN, Wang G, Guo H (2012). Predicting plant traits and functional types response to grazing in an alpine shrub meadow on the Qinghai-Tibet Plateau. Science China Earth Sciences, 55, 837-851.

责任编委: 张金屯 责任编辑: 王 蒇 